\title{
Diet-Induced Weight Loss Alters Functional Brain Responses during an Episodic Memory Task
}

\author{
Carl-Johan Boraxbekk a, b Andreas Stomby ${ }^{c} \quad$ Mats Ryberg ${ }^{c}$ \\ Bernt Lindahl $^{c}$ Christel Larsson ${ }^{d, e}$ Lars Nyberg ${ }^{b, f, g}$ Tommy Olsson ${ }^{c}$ \\ ${ }^{a}$ Center for Demographic and Aging Research, Umeå University, Umeå, Sweden; ${ }^{b}$ Umeå \\ Center for Functional Brain Imaging (UFBI), Umeå University, Umeå, Sweden; ' Public \\ Health and Clinical Medicine, Medicine, Umeå University, Umeå, Sweden; ${ }^{d}$ Department of \\ Food and Nutrition and Sport Science, University of Gothenburg, Gothenburg, Sweden; \\ e Department of Food and Nutrition, Umeå University, Umeå, Sweden; ${ }^{f}$ Integrative Medical \\ Biology, Section for Physiology, Umeå University, Umeå, Sweden; ${ }^{9}$ Radiation Sciences, \\ Section for Diagnostic Radiology, Umeå University, Umeå, Sweden
}

\section{Key Words}

Functional magnetic resonance imaging · Episodic memory · Obesity · Diet interventions · Hippocampus

\begin{abstract}
Objective: It has been suggested that overweight is negatively associated with cognitive functions. The aim of this study was to investigate whether a reduction in body weight by dietary interventions could improve episodic memory performance and alter associated functional brain responses in overweight and obese women. Methods: 20 overweight postmenopausal women were randomized to either a modified paleolithic diet or a standard diet adhering to the Nordic Nutrition Recommendations for 6 months. We used functional magnetic resonance imaging to examine brain function during an episodic memory task as well as anthropometric and biochemical data before and after the interventions. Results: Episodic memory performance improved significantly $(p=0.010)$ after the dietary interventions. Concomitantly, brain activity increased in the anterior part of the right hippocampus during memory encoding, without differences between diets. This was associated with decreased levels of plasma free fatty acids (FFA). Brain activity increased in pre-frontal cortex and superior/middle temporal gyri. The magnitude of increase correlated with waist circumference reduction. During episodic retrieval, brain activity decreased in inferior and middle frontal gyri, and increased in middle/superior temporal gyri. Conclusions: Diet-induced weight loss, associated with decreased levels of plasma FFA, improves episodic memory linked to increased hippocampal activity.


Boraxbekk et al.: Diet-Induced Weight Loss Alters Functional Brain Responses during an Episodic Memory Task

\section{Introduction}

The prevalence of obesity increases worldwide [1]. This severely affects public health due to associated comorbidities such as hypertension, type 2-diabetes, and increased cardiovascular risk [2]. Notably, overweight and obesity during midlife increases the risk of developing dementia later in life and at least one third of all Alzheimer disease (AD) cases may be attributed to modifiable risk factors including midlife obesity [3-5]. In addition to increased risk of dementia, obesity has also been associated with impaired episodic and semantic memory functions in non-demented middle-aged elderly men [6]. Importantly, there is also evidence that caloric restriction and weight loss may improve both memory and executive functions in middle-aged and elderly individuals $[7,8]$.

Imaging studies have shown that obesity also is associated with decreased global brain volume as well as decreased hippocampal volume, a key brain region for $\operatorname{AD}[9,10]$. Moreover, functional magnetic resonance imaging (fMRI) has revealed decreased activation of parietal cortex during a working memory task in obese subjects with a possible link to decreased insulin sensitivity [11]. In line with behavioral data, there is support that weight loss may alter brain response. A recent study suggests that obese individuals have increased glucose metabolism in posterior cingulate gyrus compared to normal-weight persons and that this can be reversed by weight loss after gastric bypass [12]. However, there are no studies evaluating effects of diet-induced weight loss on functional brain response during episodic memory testing. This is of major interest since it may indicate ways to improve hippocampus function and thereby lowering AD risk in obese individuals.

Notably, specific nutrients may influence brain functioning. A high intake of saturated fatty acids in rodents is associated with impaired neurogenesis and dendritic integrity as well as with increased inflammation in the hippocampus linked to impaired memory functions $[13,14]$. In humans, a high intake of saturated fatty acids was associated with decreased cognitive performance over a 6-year period [15]. In contrast, several cohort studies have found that close adherence to a Mediterranean diet, with a high intake of unsaturated fatty acids, may reduce risk of developing mild cognitive impairment and AD [16]. Thus both experimental and epidemiological research suggests important links between brain function and fatty acid balance.

In summary, there is a need for studies on the underlying neural mechanisms for memory improvement following weight loss. Our main objective was to measure functional brain response during an episodic memory task before and after 6 months of ad libitum dietary interventions. The second aim was to investigate whether a paleolithic diet or a standard diet according to Nordic Nutrition Recommendations had different effects on functional brain response in overweight and obese postmenopausal women.

\section{Participants and Methods}

\section{Study Participants}

This study was part of a larger project investigating anthropometric and metabolic effects of two different diets consumed ad libitum, either a modified paleolithic-type diet (PD) or a standard diet according to the Nordic Nutrition Recommendations (NNR) (see Mellberg et al. [17]). 20 females that were included in the diet intervention during the same time period were offered, and agreed, to take part in the functional brain imaging study (mean age 61.3 years, range 52-69 years). 11 subjects were randomized to the NNR diet and 9 to the PD. All participants were postmenopausal ( $>1$ year since last menstruation), overweight or obese (BMI 27-40 kg/m²), without self-reported cognitive impairments (table 1). Exclusion criteria were history of diabetes, consumption of a restricted diet, previous heart disease, or any medication for psychiatric disorders [17]. 
Boraxbekk et al.: Diet-Induced Weight Loss Alters Functional Brain Responses during an Episodic Memory Task

Table 1. Anthropometric measurements and biochemical data (mean and SD) of postmenopausal women ( $\mathrm{n}=20)$ at baseline and after 6 months of dietary interventions

\begin{tabular}{|c|c|c|c|c|}
\hline & \multicolumn{2}{|l|}{$\begin{array}{l}\text { PD group } \\
(\mathrm{n}=9)\end{array}$} & \multicolumn{2}{|c|}{$\begin{array}{l}\text { NNR diet group } \\
(n=11)\end{array}$} \\
\hline & baseline & 6 months & baseline & 6 months \\
\hline Age, years & $61.1(1.6)$ & & $61.6(1.7)$ & \\
\hline Weight, kg** & $84.8(5.6)$ & $75.1(5.8)$ & $85.1(4.8)$ & $79.1(4.9)$ \\
\hline BMI, $\mathrm{kg} / \mathrm{m}^{2 * *}$ & $31.9(1.7)$ & $28.3(2.0)$ & $32.4(2.4)$ & $30.1(2.2)$ \\
\hline Waist, $\mathrm{m}^{* *}$ & $0.90(0.05)$ & $0.86(0.05)$ & $0.91(0.09)$ & $0.88(0.07)$ \\
\hline Plasma FFAs, mmol/la* & $0.67(0.14)$ & $0.54(0.10)$ & $0.58(0.21)$ & $0.53(0.18)$ \\
\hline HOMA-IR & $2.0(1.1)$ & $1.7(0.7)$ & $2.2(1.2)$ & $2.0(1.1)$ \\
\hline Plasma glucose, $\mathrm{mmol} / \mathrm{l}$ & $5.1(1.0)$ & $5.2(0.6)$ & $4.9(0.6)$ & $5.4(1.4)$ \\
\hline Plasma insulin, mIU/l & $8.6(3.9)$ & $7.3(2.5)$ & $9.8(4.7)$ & $8.1(3.7)$ \\
\hline PAEE, kcal/day & $686(244)$ & $782(364)$ & $853(243)$ & $811(232)$ \\
\hline Energy intake, kcal/day** & $1,941(341)$ & $1,698(235)$ & $2,193(426)$ & $1,676(364)$ \\
\hline Protein, E\%** & $17(2)$ & $24(2)^{\#}$ & $17(2)$ & $19(1)$ \\
\hline Fat, E\%* & $33(4)$ & $44(4)^{\#}$ & $33(4)$ & $30(6)$ \\
\hline Carbohydrate, E\%** & $46(3)$ & $28(3)^{\#}$ & $45(4)$ & $45(6)$ \\
\hline MUFA:SFA** & $1.0(0.19)$ & $2.4(0.4)^{\#}$ & $1.0(0.2)$ & $1.2(0.2)$ \\
\hline PUFA:SFA*** & $0.4(0.2)$ & $1.1(0.4)^{\#}$ & $0.4(0.1)$ & $0.6(0.2)$ \\
\hline
\end{tabular}

MUFA:SFA = ratio of monounsaturated fatty acids to saturated fatty acids; PUFA:SFA = ratio of polyunsaturated fatty acids to saturated fatty acids.

${ }^{a}$ For plasma FFAs $n=9$ in the PD group and $n=10$ in the NNR diet group.

Time effect: ${ }^{*} \mathrm{p}<0.01{ }^{* *} \mathrm{p}<0.001,{ }^{* * *} \mathrm{p}<0.001$.

\#Diet $\times$ time effect.

\section{Diet Intervention}

A detailed description of the complete dietary intervention was recently published [17]. Briefly, the PD aimed to provide 30 energy percent (E\%) as protein, $40 \mathrm{E} \%$ as fat, and $30 \mathrm{E} \%$ as carbohydrates, with a recommendation of a high intake of mono- and polyunsaturated fatty acids (MUFAs and PUFAs), and was based on foods such as lean meat, fish, fruit, vegetables, root vegetables, eggs, and nuts. The NNR diet was lower in protein and fat content and higher in carbohydrates, aiming for a daily intake of $15 \mathrm{E} \%$ protein, 25-30 E\% fat, and 55-60 E\% carbohydrates with emphasis on low-fat dairy and high-fiber products [18]. Participants met with a dietician eight times (group sessions) during the first 6 months of the intervention. The sessions contained cooking of intervention diets, information about dietary effects on health, how to change behavior, and group discussions. Notably, both diets were ad libitum without any restrictions in total calorie intake. Dietary intake was assessed using 4-day self-reported food records at baseline and at 6 months. The protocol was in accordance with the Helsinki declaration, and approved by the Regional Ethical Review Board at Umeå University, Sweden. Written informed consent was obtained from all participants. This trial was registered at clinicaltrials.gov as NCT00692536.

\section{Anthropometric Measurements and Physical Activity Estimation}

All measurements were made at baseline and 6 months after beginning the intervention. The participants were weighed in light indoor clothing without shoes to the nearest $0.1 \mathrm{~kg}$ on a calibrated electronic digital scale. Height was measured to the nearest $\mathrm{cm}$. BMI was calculated as weight $/ \mathrm{lengt \textrm {h } ^ { 2 }}\left(\mathrm{kg} / \mathrm{m}^{2}\right)$. Waist circumference was measured midway between the lower rib margin and the iliac crest during gentle exhalation. To control for physical activity as a confounding factor, physical activity energy expenditure (PAEE) was assessed using individually combined accelerometry and heart rate monitoring for 7 consecutive days, calculated as described previously [17].

\section{Biochemical Data}

Fasting venous blood samples were drawn at baseline and after 6 months. After analysis of plasma insulin (Elecsys insulin kit on a Modular E170 immunoanalyzer, Roche Diagnostics, Indianapolis, IN, USA) 
Boraxbekk et al.: Diet-Induced Weight Loss Alters Functional Brain Responses during an Episodic Memory Task

and glucose levels (Vitros Slides on a Vitros 5.1FS, Ortho-clinical Diagnostics, Johnson \& Johnson, New Brunswick, NJ, USA), the homeostasis model assessment for insulin resistance (HOMA-IR) was used to estimate insulin resistance ((fasting glucose $\times$ fasting insulin) / 22.5). Plasma free fatty acid (FFA) levels were analyzed in fasting blood samples (NEFA-HR2, Wako Chemicals, Neuss, Germany).

\section{fMRI Parameters}

T2*-weighted images were acquired using high-speed echoplanar imaging (EPI) sequence on a Philips 3T scanner. Ten dummy scans were performed prior to image acquisition to avoid saturation artifacts. Parameters used were: TR $=1,512 \mathrm{~ms} ; 31$ slices; TE $=30 \mathrm{~ms}$; flip angle $70^{\circ}$; field of view 22T22cm; 64264 matrix; $3.4463 .44 \mathrm{~mm}$ in-plane $34.65 \mathrm{~mm}$ thick; 8-channel SENSE head coil with a SENSE-factor of 2.6. The images were pre-processed using the following steps: realignment and unwarping, slice-timing correction, normalization (to MNI space), and smoothed using an $8.0 \mathrm{~mm}$ FWHM Gaussian kernel. Pre-processing and data analysis were completed using SPM8 (Wellcome Department of Cognitive Neurology, London, UK).

\section{fMRI Task}

A face-name paradigm was used to examine brain responses related to episodic memory. This paradigm has previously been shown to engage brain regions underlying episodic memory, such as the hippocampus and the prefrontal cortex [19]. The task consisted of encoding, retrieval, and a control sequence. Inside the scanner, a mirror was attached to the head coil allowing the participant to watch a computer screen onto which stimulus materials were presented using E-prime 1.1 (Psychology Software Tools, Sharpsburg, PA, USA). During encoding, a fictional name and an unknown face were presented on the screen. The participants were instructed to remember the name associated with each face, and press a button with their right hand as soon as they have memorized the face-name pair. The same faces that appeared during encoding were presented again during retrieval. However, instead of showing the name associated with the face, three different letters were presented next to each face. The task was to indicate which of the three letters corresponded to the first letter of the name that was associated with the face. One correct alternative was always provided. The participants responded by pressing a button with their right hand. If the first letter was correct they pressed with the index finger, if the second letter was correct they pressed with the middle finger, and if the third letter was correct they used the ring finger. The encoding and retrieval stimuli were presented for $4 \mathrm{~s}$ with $1 \mathrm{~s}$ interstimulus interval. For the baseline task, a fixation cross was presented on the screen for 1,500-2,500 ms. This cross was replaced, randomly, with a circle for $500 \mathrm{~ms}$. When this occurred the participants were instructed to press any button as quickly as possible. The circle was then followed by another fixation cross for 2,000-3,000 ms, making the total presentation time to $5 \mathrm{~s}$. A blocked design was used with six blocks of encoding and retrieval and eight blocks of baseline alternated throughout the experiment. Each block was $20 \mathrm{~s}$ long for the encoding, retrieval, and the baseline task. There were a total of 24 face-name pairs. The same procedure with the same faces and names was performed before and after 6 months of dietary interventions.

\section{Data Analysis}

After pre-processing, single subject contrasts were set up using the general linear model with the different conditions (encoding, retrieval, baseline) as separate regressors. Group-averaged data were analyzed in a random-effects model. First the participants were analyzed as one group to examine the effects on brain function before versus after diet-induced weight loss. The first step in the analysis was to generate statistical parametric maps (SPMs) using repeated measures ANOVA, in order to reveal changes in brain regions between the pre- and post-diet data collections for encoding and retrieval. The main contrasts for this analysis were (encoding>baseline) and (retrieval>baseline). Thereafter, individual BOLD signal change relative to mean-of-session activity calculated as $\left(\left(\beta_{\text {Encoding }}-\beta_{\text {Baseline }}\right) / \beta_{\text {Constant }}\right) \times 100$ and $\left(\left(\beta_{\text {Retrieval }}-\right.\right.$ $\beta_{\text {Baseline }}$ ) $\left./ \beta_{\text {Constant }}\right) \times 100$ was extracted from each significant peak of activation in order to analyze potential differences between the PD and standard diet according to NNR using ANOVAs.

Due to the link between episodic memory impairment and hippocampal damage in AD, we made specific analyses of this brain region. Regions of interests (ROI) were selected at two different parts of the hippocampus (anterior and posterior portion), bilaterally. The selection was based on the findings by Salami et al. [20]. By using the same face-name paradigm, Salami et al. [20] concluded that these four clusters were highly activated and important for memory function during the used memory task. The ROIs were established based on anatomical localization using the Talairach and Tournoux [21] brain atlas. Each ROI was defined as a sphere of $5 \mathrm{~mm}$ diameter with the center of the sphere at the defined coordinate. Each ROI value was extracted 
Boraxbekk et al.: Diet-Induced Weight Loss Alters Functional Brain Responses during an Episodic Memory Task

by computing the brain activity (beta values) during encoding or retrieval in relation to the mean activity during the session. This generated single subject values for each ROI during encoding and retrieval, before and after dietary interventions.

Memory performance during scanning as well as anthropometric measures and biochemistry were analyzed using repeated measures ANOVA to compare performance before and after the dietary interventions. We also examined the shared variability $\left(R^{2}\right)$ between significant changes of brain activity for each peak of activation and changes in anthropometric and biochemical data (BMI, waist circumference, HOMA, FFA levels) as well as in memory performance.

In the SPM analysis, we applied a statistical threshold of $\mathrm{p}<0.05$, FWE correction for multiple comparisons, with a cluster extent larger than 10 voxels. For ANOVAS on memory performance, anthropometrics/ biochemistry, and correlations, $\mathrm{p}<0.05$ (uncorrected) was considered statistically significant.

Reference Sample

As a reference sample we used data from a recent population-based study of cognitive function from our research site $(n=147)$. Importantly, this study used the same face-name paradigm [19]. Since we had access to original data from Pudas et al. [19], we compared each peak from the brain regions displayed as significant change in the present study in relation to the encoding and retrieval network from the reference sample.

\section{Results}

Anthropometric Measurements, Biochemistry, and Dietary Intake

Despite the ad libitum approach, both the PD and standard diet according to NNR improved anthropometric measurements after 6 months without significant differences between groups. The self-reported total energy intake decreased significantly in both groups, without any group differences. However, self-reported intake of protein, fat, and the ratio of MUFA and PUFA to saturated fatty acids increased, whereas the carbohydrate intake decreased significantly in the PD group compared to the NNR diet group. Levels of FFA in plasma decreased in both groups, but there were no effects on plasma glucose, insulin or HOMA-IR. PAEE was unaltered throughout the study period (table 1).

\section{Memory Performance}

Memory performance improved significantly from $16.7 \pm 3.3$ correctly remembered facename pair, out of maximum 24 , at baseline to $18.5 \pm 2.0$ after the dietary interventions, $\mathrm{F}(1$, $18)=8.3 ; p=0.010$; without difference between the PD or standard diet according to NNR.

\section{Brain Imaging Data}

Significant changes (baseline vs. 6 months) in brain activation during the encoding phase were observed throughout the brain without differences between the PD and standard diet according to NNR (fig. 1A). The further analyses therefore include both study groups. In middle superior frontal gyrus and superior temporal gyrus, functional brain activity increased after the dietary interventions (fig. 1A). Decreased waist circumference correlated significantly with increased brain activation in the superior temporal gyrus BA $22\left(-50-142 ; R^{2}=0.36, p=0.006\right)$ and in the insula $\left(-38-20-2 ; R^{2}=0.25 ; p=0.028\right)$. The fusiform face area in the ventral occipito-temporal cortex [22] showed decreased activation, which was significantly correlated with memory improvements; $R^{2}=0.20, p=0.049$. This brain region was also described in the reference sample as part of the encoding network for this task [19]. Decreased levels of FFA correlated with increased brain activation in the fusiform gyrus (44-60-14; $\mathrm{R}^{2}=0.30, \mathrm{p}=0.016$ ) however; when two outliers $( \pm 2 \mathrm{SD}$ in changed brain activity) were removed the correlation disappeared $\left(R^{2}=0.03, p=0.53\right)$. 
Boraxbekk et al.: Diet-Induced Weight Loss Alters Functional Brain Responses during an Episodic Memory Task

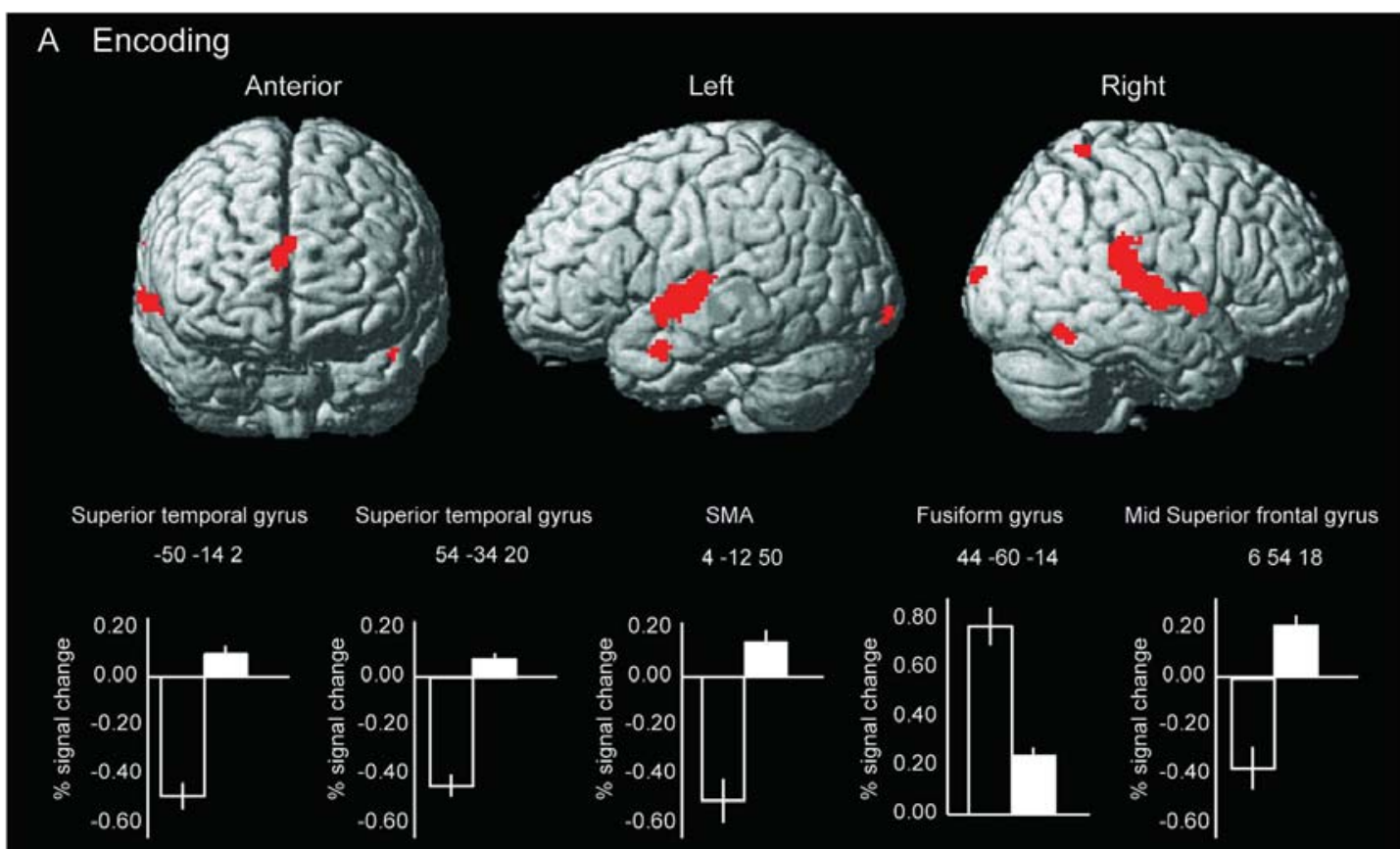

B Retrieval

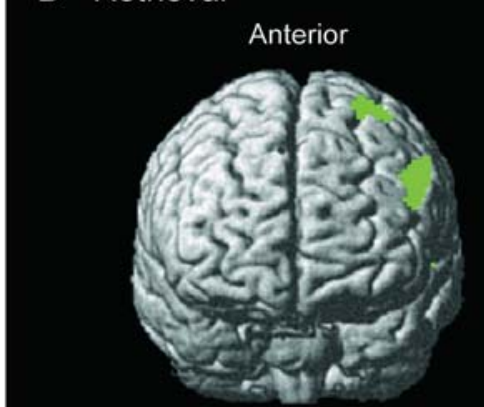

Inferior frontal gyrus

$-40430$

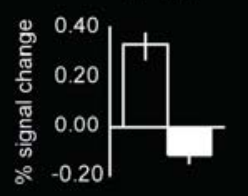

Associate visual cortex

$$
30-7040
$$

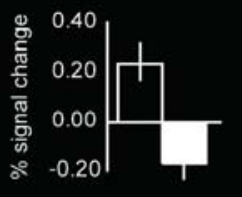

Left

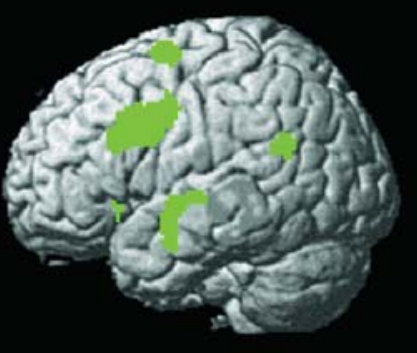

Right

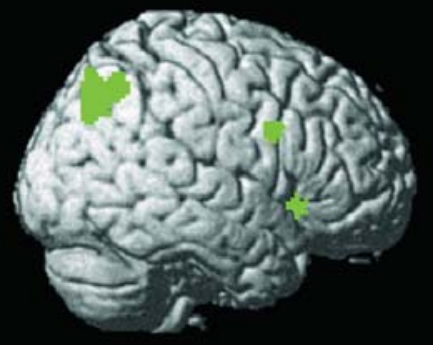

Inferior frontal gyrus

$3220-6$

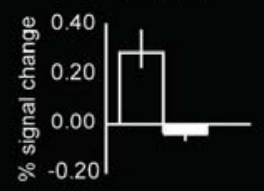

Middle temporal gyrus

$-52-6-6$

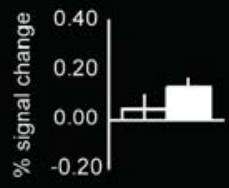

Superior temporal gyrus $-42-22-2$

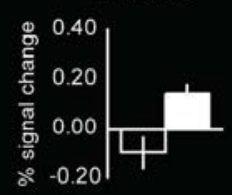

Fig. 1. Brain regions showing significant changes ( $p<0.05$; FWE-corrected for multiple comparisons) in functional brain response during memory encoding (A) and retrieval (B) after diet-induced weight loss in postmenopausal overweight and obese women $(n=20)$. Functional brain data is overlaid onto a rendered standardized brain in MNI space. For each cluster, the strongest peak (x y z coordinates) is plotted as bars (with standard errors) indicating \% BOLD signal change in relation to mean of session pre (black bars) and post (white bars) the dietary interventions. 
Boraxbekk et al.: Diet-Induced Weight Loss Alters Functional Brain Responses during an Episodic Memory Task

Table 2. Brain regions associated with significant change (FWE; $p<0.05$ ) of activation during the encoding and retrieval phases of an episodic memory task after 6-month dietary interventions in postmenopausal overweight and obese women $(n=20)$

\begin{tabular}{|c|c|c|c|c|c|c|c|}
\hline Direction of change & Region & BA & $\mathrm{x}$ & $\mathrm{y}$ & $\mathrm{z}$ & $\mathrm{k}$ & Z-value \\
\hline \multicolumn{8}{|l|}{ Encoding } \\
\hline Increase & superior temporal gyrus & 22 & -50 & -14 & 2 & 336 & 6.1 \\
\hline Increase & Insula & & -38 & -20 & -2 & & 5.5 \\
\hline Increase & superior temporal gyrus & 42 & 54 & -34 & 20 & 358 & 5.8 \\
\hline Increase & superior temporal gyrus & 22 & 60 & -2 & 0 & & 5.2 \\
\hline Increase & SMA & 6 & 4 & -12 & 50 & 71 & 5.4 \\
\hline Decrease & fusiform gyrus & 37 & 44 & -60 & -14 & 22 & 5.3 \\
\hline Increase & middle superior frontal gyrus & $9 / 10$ & 6 & 54 & 18 & 12 & 5.0 \\
\hline \multicolumn{8}{|l|}{ Retrieval } \\
\hline Decrease & inferior frontal gyrus & 44 & -40 & 4 & 30 & 281 & 6.1 \\
\hline Decrease & middle frontal gyrus & 9 & -46 & 18 & 28 & & 5.6 \\
\hline Decrease & associate visual cortex & 19 & 30 & -70 & 40 & 138 & 5.9 \\
\hline Decrease & inferior frontal gyrus (anterior insula) & 47 & 32 & 20 & -6 & 30 & 5.5 \\
\hline Increase & middle temporal gyrus & 21 & -52 & -6 & -6 & 17 & 5.1 \\
\hline Increase & middle temporal gyrus & 21 & -50 & -2 & -16 & & 4.7 \\
\hline Increase & superior temporal gyrus & 21 & -42 & -22 & -2 & 12 & 5.0 \\
\hline
\end{tabular}

Table 2 comprises a complete list of effects, with exact locations (indicated as $\mathrm{x} y \mathrm{z}$ coordinates in the MNI space), cluster extent, and Z-values.

For the retrieval phase (fig. $1 \mathrm{~B}$ ), brain activity decreased in the inferior and middle frontal gyri after the dietary interventions. These regions were described as part of the retrieval network from Pudas et al. [19]. Further, the middle and superior temporal gyri increased in activity (table 2). There were no differences between the two diets, and no association between changes in brain activity and anthropometric or biochemical variables was found.

\section{ROI Analysis of Hippocampus}

At 6 months, the anterior part of the right hippocampus exhibited increased activity during the encoding task $(F(1,18)=6.08, p<0.05)$, without differences between diet groups. Notably, the increased hippocampal activity was associated with decreased levels of plasma FFA $\left(\mathrm{R}^{2}=0.27, \mathrm{p}=0.024\right.$; fig. 2$)$. There were no other correlations between changes in hippocampus activity and anthropometric or biochemical measures. There were no significant changes in activation in the right posterior or left anterior/posterior parts of the hippocampus.

\section{Discussion}

The main finding in our study is that memory improvement may occur after a dietinduced weight loss via ad libitum food intake that concomitantly alters functional brain response of an episodic memory task. Importantly, increased brain activity in the anterior part of the right hippocampus was seen during memory encoding. Taken together, this suggests a more efficient brain function that potentially may be translated into a reduced dementia risk given that the hippocampal region is in focus for neurodegenerative processes linked to AD. 
Fig. 2. Changes in hippocampal activity and plasma FFAs after diet-induced weight loss, $\mathrm{R}^{2}=0.27$, $\mathrm{p}=0.024$. Note, $\mathrm{n}=19$ in this analysis due to a missing blood sample for one of the participants in the NNR diet group.

Boraxbekk et al.: Diet-Induced Weight Loss Alters Functional Brain Responses during an Episodic Memory Task

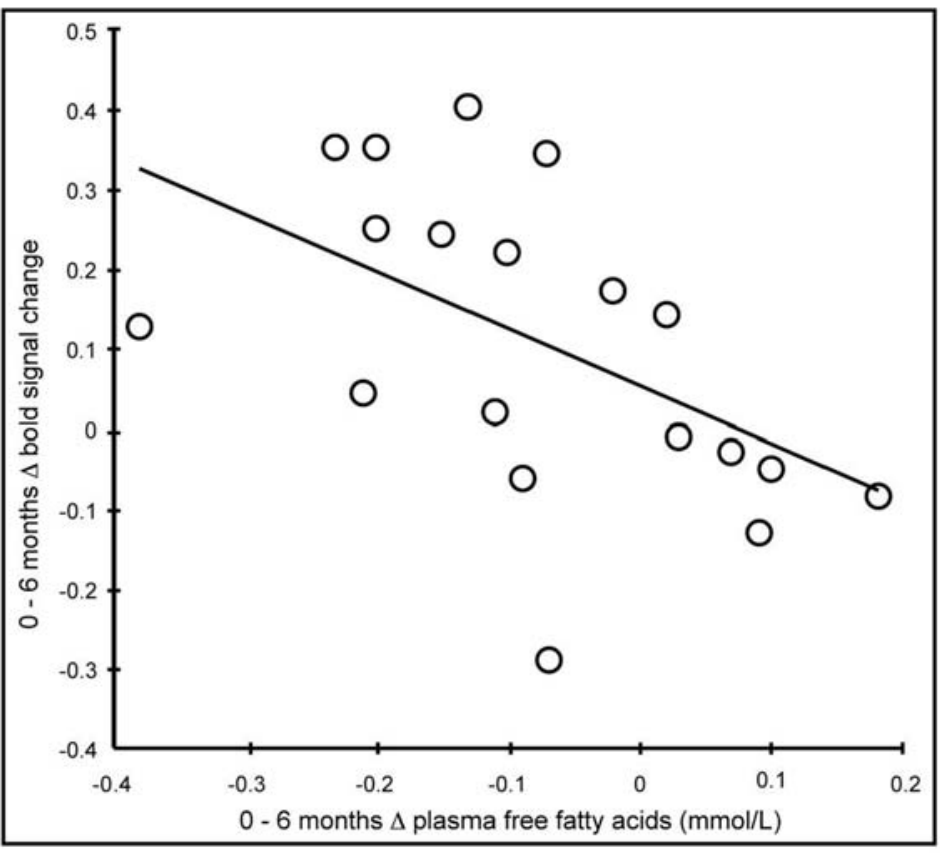

Recently, using positron emission tomography to measure brain metabolism, it was shown that severely obese (BMI $>50 \mathrm{~kg} / \mathrm{m}^{2}$ ) individuals improved performance on a working memory task accompanied by a normalization of brain metabolism after gastric bypass weight loss [12]. This is in line with our data, suggesting an improved memory after dietinduced weight loss. We were also able to extend these findings by analyzing detailed functional responses. The increased activity in the anterior part of the right hippocampus during memory encoding in our study is thus a novel finding that may provide a first indication of the neural basis for increased memory performance and lowered risk of dementia after weight loss [5, 7]. Previous studies have shown that 6 months to 1 year of aerobic exercise increased hippocampal volume and improved memory performance in sedentary elderly participants [23]. Since the participants did not increase their physical activity during the study, we consider this as a unique effect related to weight loss caused by a diet change. In the present study we were not able to analyze potential changes of hippocampus volume, which certainly would be of interest for future studies. Notably, the memory performance improved significantly as well, albeit not correlated to hippocampal activation. However, in a previous study using the same face-name paradigm the BOLD response in right anterior hippocampus was correlated to memory performance [20].

Decreased functional brain response in the fusiform face area during memory encoding was associated with memory improvements after the diet interventions. This region, together with the hippocampus, is part of the encoding network presented by Pudas et al. [19]. The fusiform face area is specialized for facial recognition [22], and reduced activity in this region, together with improved memory performance, may also reflect more efficient encoding after the dietary interventions. We also found that decreased waist circumference was associated with increased brain activation in the insula and the superior temporal cortex (BA 48 and 22) during the encoding phase of the episodic memory task. The insula region has previously been suggested as a key region for recognition memory [24] as well as for cognitive control [25]. Further, the superior temporal cortex has been suggested as a region activated by expressive faces [26], and together with insula it is used for identification and matching [27]. 
Boraxbekk et al.: Diet-Induced Weight Loss Alters Functional Brain Responses during an Episodic Memory Task

Activity increases, albeit not correlated with anthropometry, were also present in the superior frontal gyrus (BA 9/10), a region previously associated with self-referential activity and emotional processing [28].

Taken together, the changes in brain activation suggest that the women in this study were able to recruit brain resources more efficiently after the dietary interventions. This may have allowed them to identify relevant information to encode, which may have resulted in a deeper and more efficient consolidation.

During retrieval, brain activity increased in the superior and middle temporal gyri, regions that previously have been associated with recognition of famous faces retrieved from long-term memory [29]. Decreases in brain activity following the dietary interventions were located in inferior and middle frontal gyrus, which has been associated with retrieval of episodic memories [30], and BA 47, which has previously been related with retrieval effort [31]. Cognitive demanding tasks usually involve recruiting frontal regions of the brain [32]. The decreased activity in pre-frontal cortical regions could thus be interpreted as if the task was less cognitively demanding after the interventions. This is in line with findings from memory training studies reporting reduced frontal activity in relation to improved memory function after intervention [33]. The regions displaying activity decreases were also reported in the reference sample [19].

One objective was to test if the PD and standard diet according to NNR had different effects on memory performance and functional brain response. Although the PD group reported a significantly altered dietary intake compared with the NNR diet group, we did not find any differences in memory performance or functional brain responses between the two diets. This may be due to both lack of power to detect such differences as well as limited duration of the intervention. Notably, the improvements in anthropometric measurements, insulin sensitivity, and FFA levels were similar in both diet groups, which may also attenuate group differences. Therefore, no firm conclusion regarding diet-specific effects could be drawn from this study.

Interestingly, decreased plasma FFA levels were associated with increased hippocampus activity. In rodents, saturated FFA can induce inflammation in hypothalamus via activation of toll-like receptor 4, which may influence appetite regulation [34]. In addition, 20 weeks of a high-fat diet causes hippocampal inflammation that is reversed by treatment with the antioxidant resveratrol in mice [35], which is in line with a recent study on humans suggesting that resveratrol treatment improves memory and functional connectivity of the hippocampus [36]. Thus, we propose that a potential underlying mechanism linking the dietary interventions to improved hippocampus function may be that decreased levels of FFA leads to reduced hippocampal inflammation. In further support of this mechanism decreased levels of FFA correlated with decreased levels of C-reactive protein (data not shown).

There are methodological limitations that should be considered. The anthropometric and biochemical effects were similar in both the standard diet group and the PD group. Thus, we did not include a weight-stable observational group in this study, but used data from an extensive population-based study on cognitive function performed with the same fMRI paradigm within the same research site [19]. Therefore, we could control that the brain responses were within the encoding and retrieval brain network characterized in this population. The effects of the dietary interventions presented here could also, in part, reflect testretest effects unrelated to the specific intervention. Notably, a similar episodic memory facename paradigm is associated with good test-retest reliability [37]. In addition, slight reductions of brain activity may occur as a function of re-testing [38]. In contrast, increased brain activity during encoding such as in the hippocampus in our study most likely reflects effects of the diet interventions. It should also be noted that the sample size in this study was small, limiting the possibility to find group differences, and none of the correlations presented 
'survived' a Bonferroni correction for multiple comparisons. In addition, the improved memory performance in our study could be influenced by training effects since the same memory task was provided at both baseline and 6 months. Considering the limitations of the present study, these results require further validation with inclusion of an observational study group without dietary changes.

It would be of interest to include both women and men in future studies since the association between obesity and impaired cognitive functions [6] as well as the risk of dementia [39] may be gender-specific. Individuals with different metabolic backgrounds should also be studied, especially since the risk of AD increases substantially with the development of type 2 diabetes [40]. In addition, possible diet and gene interaction factors, such as the APOE4 genotype, should also be taken into consideration in future studies [41].

\section{Conclusions}

Diet-induced weight loss in overweight postmenopausal women increases hippocampal activity during memory encoding. This effect may be linked to decreased levels of plasma FFA and provide a potential underlying mechanism why weight loss may improve memory performance and decrease risk of developing dementia.

\section{Trial Registration}

This trial was registered at clinicaltrials.gov as NCT00692536

\section{Author Contributions}

TO and LN designed research, CJO collected MRI data, CJO and AS analyzed data. All authors were involved in the writing of the paper and had final approval of the submitted and published versions.

\section{Acknowledgments}

We wish to thank all of the participants for taking part in this study. We also wish to thank Susanne Sandberg, Caroline Mellberg (Public Health and Clinical Medicine, Umeå University), Sören Brage (MRC Epidemiology Unit, University of Cambridge, United Kingdom), and Sara Pudas (Integrative Medical Biology, Umeå University, Sweden) for valuable scientific contributions. This project was supported by the Swedish Research Council, the Swedish Heart and Lung Foundation, the King Gustaf V and Drottning Victoria Foundation, Umeå University, the Northern Sweden County Councils, the Västerbotten County Council, the Swedish Council for Working Life and Social Research (2006-0699 and 2010-0398), and the Knut and Alice Wallenberg Foundation.

\section{Disclosure Statement}

On behalf of all authors, the corresponding author states that there is no conflict of interest. 
Boraxbekk et al.: Diet-Induced Weight Loss Alters Functional Brain Responses during an Episodic Memory Task

\section{References}

1 Finucane MM, Stevens GA, Cowan MJ, Lin JK, Paciorek CJ, Gitanjali MS, Gutierrez HR, Lu Y, Bahalim AN, Farzadfar F, Riley LM, Ezzati M: National, regional, and global trends in body-mass index since 1980: systematic analysis of health examination surveys and epidemiological studies with 960 country-years and $9 \cdot 1$ million participants. Lancet 2011;377:557-567.

2 Visscher TLS, Seidell JC: The public health impact of obesity. Annu Rev Public Health 2001;22:355-375.

-3 Xu WL, Atti AR, Gatz M, Pedersen NL, Johansson B, Fratiglioni L: Midlife overweight and obesity increase latelife dementia risk: a population-based twin study. Neurology 2011;76:1568-1574.

4 Whitmer RA, Gunderson EP, Barrett-Connor E, Quesenberry CP, Yaffe K: Obesity in middle age and future risk of dementia: a 27 year longitudinal population based study. BMJ 2005;330:1-5.

-5 Norton S, Matthews FE, Barnes DE, Yaffe K, Brayne C: Potential for primary prevention of Alzheimer's disease: an analysis of population-based data. Lancet Neurol 2014;13:788-794.

6 Elias MF, Elias PK, Sullivan LM, Wolf PA, D'Agostino RB: Lower cognitive function in the presence of obesity and hypertension: the Framingham Heart Study. Int J Obes 2003;27:260-268.

-7 Siervo M, Arnold R, Wells JCK, Tagliabue A, Colantuoni A, Albanese E, Brayne C, Stephan BCM: Intentional weight loss in overweight and obese individuals and cognitive function: a systematic review and metaanalysis. Obes Rev 2011;12:968-983.

-8 Witte A V, Fobker M, Gellner R, Knecht S, Flöel A: Caloric restriction improves memory in elderly humans. Proc Natl Acad Sci U S A 2009;106:1255-1260.

-9 Gunstad J, Paul RH, Cohen RA, Tate DF, Spitznagel MB, Grieve S: Relationship between body mass index and brain volume in healthy adults. Int J Neurosci 2008;118:1582-1593.

-10 Raji CA, Ho AJ, Parikshak NN, Becker JT, Lopez OL, Kuller LH, Hua X, Leow AD, Toga AW, Thompson PM: Brain structure and obesity. Hum Brain Mapp 2010;31:353-364.

11 Gonzales MM, Tarumi T, Miles SC, Tanaka H, Shah F, Haley AP: Insulin sensitivity as a mediator of the relationship between BMI and working memory-related brain activation. Obesity (Silver Spring) 2010;18:21312137.

12 Marques EL, Halpern A, Corrêa Mancini M, de Melo ME, Horie NC, Buchpiguel CA, Coutinho AMN, Ono CR, Prando S, Santo MA, Cunha-Neto E, Fuentes D, Cercato C: Changes in neuropsychological tests and brain metabolism after bariatric surgery. J Clin Endocrinol Metab 2014;99:E2347-2352.

13 Lindqvist A, Mohapel P, Bouter B, Frielingsdorf H, Pizzo D, Brundin P, Erlanson-Albertsson C: High-fat diet impairs hippocampal neurogenesis in male rats. Eur J Neurol 2006;13:1385-1388.

14 Granholm A-C, Bimonte-Nelson HA, Moore AB, Nelson ME, Freeman LR, Sambamurti K: Effects of a saturated fat and high cholesterol diet on memory and hippocampal morphology in the middle-aged rat. J Alzheimers Dis 2008;14:133-145.

-15 Morris MC, Evans DA, Bienias JL, Tangney CC, Wilson RS: Dietary fat intake and 6-year cognitive change in an older biracial community population. Neurology 2004;62:1573-1579.

16 Singh B, Parsaik AK, Mielke MM, Erwin PJ, Knopman DS, Petersen RC, Roberts RO: Association of mediterranean diet with mild cognitive impairment and Alzheimer's disease: a systematic review and meta-analysis. J Alzheimers Dis 2014;39:271-282.

17 Mellberg C, Sandberg S, Ryberg M, Eriksson M, Brage S, Larsson C, Olsson T, Lindahl B: Long-term effects of a palaeolithic-type diet in obese postmenopausal women: a 2-year randomized trial. Eur J Clin Nutr 2014;68: 1350-1357.

18 Becker W, Lyhne N, Pedersen AN, Aro A, Fogelholm M, Thorsdottir I, Alexander J, Andersson SA, Meitzer HM, Pedersen JI: Nordic Nutrition Recommendations 2004. Integrating nutrition and physical activity. Scand J Nutr 2004;48:178-187.

19 Pudas S, Persson J, Josefsson M, de Luna X, Nilsson L-G, Nyberg L: Brain characteristics of individuals resisting age-related cognitive decline over two decades. J Neurosci 2013;33:8668-8677.

20 20. Salami A, Eriksson J, Nyberg L: Opposing effects of aging on large-scale brain systems for memory encoding and cognitive control. J Neurosci 2012;32:10749-10757.

21 Talairach J, Tournoux P: Co-Planar Stereotaxic Atlas of the Human Brain. New York, Thieme, 1988.

-22 Kanwisher N, McDermott J, Chun MM: The fusiform face area: a module in human extrastriate cortex specialized for face perception. J Neurosci 1997;17:4302-4311.

23 Erickson KI, Voss MW, Prakash RS, Basak C, Szabo A, Chaddock L, Kim JS, Heo S, Alves H, White SM, Wojcicki TR, Mailey E, Vieira VJ, Martin SA, Pence BD, Woods JA, McAuley E, Kramer AF: Exercise training increases size of hippocampus and improves memory. Proc Natl Acad Sci U S A 2011;108:3017-3022.

24 Bermudez-Rattoni F, Okuda S, Roozendaal B, McGaugh JL: Insular cortex is involved in consolidation of object recognition memory. Learn Mem 2005;12:447-449.

25 Dosenbach NUF, Fair DA, Miezin FM, Cohen AL, Wenger KK, Dosenbach RAT, Fox MD, Snyder AZ, Vincent JL, Raichle ME, Schlaggar BL, Petersen SE: Distinct brain networks for adaptive and stable task control in humans. Proc Natl Acad Sci U S A 2007;104:11073-11078.

-26 Britton JC, Taylor SF, Sudheimer KD, Liberzon I: Facial expressions and complex IAPS pictures: common and differential networks. Neuroimage 2006;31:906-919.

27 Mizelle JC, Wheaton LA: Neural activation for conceptual identification of correct versus incorrect tool-object pairs. Brain Res 2010;1354:100-112. 
Boraxbekk et al.: Diet-Induced Weight Loss Alters Functional Brain Responses during an Episodic Memory Task

28 Gusnard DA, Akbudak E, Shulman GL, Raichle ME: Medial prefrontal cortex and self-referential mental activity: Relation to a default mode of brain function. Proc Natl Acad Sci U S A 2001;98:4259-4264.

29 Leveroni CL, Seidenberg M, Mayer AR, Mead LA, Binder JR, Rao SM: Neural systems underlying the recognition of familiar and newly learned faces. J Neurosci 2000;20:878-886.

-30 Ranganath C, Johnson MK, Esposito MD: Left anterior prefrontal activation increases with demands to recall specific perceptual information. J Neurosci 2000;20:1-5.

31 Cabeza R, Nyberg L: Imaging cognition II: an empirical review of 275 PET and fMRI studies. J Cogn Neurosci 2000;12:1-47.

-32 Duncan J, Owen AM: Common regions of the human frontal lobe recruited by diverse cognitive demands. Trends Neurosci 2000;23:475-483.

33 Dahlin E, Neely AS, Larsson A, Bäckman L, Nyberg L: Transfer of learning after updating training mediated by the striatum. Science 2008;320:1510-1512.

34 Milanski M, Degasperi G, Coope A, Morari J, Denis R, Cintra DE, Tsukumo DML, Anhe G, Amaral ME, Takahashi HK, Curi R, Oliveira HC, Carvalheira JBC, Bordin S, Saad MJ, Velloso LA: Saturated fatty acids produce an inflammatory response predominantly through the activation of TLR4 signaling in hypothalamus: implications for the pathogenesis of obesity. J Neurosci 2009;29:359-370.

-35 Jeon BT, Jeong EA, Shin HJ, Lee Y, Lee DH, Kim HJ, Kang SS, Cho GJ, Choi WS, Roh GS: Resveratrol attenuates obesity-associated peripheral and central inflammation and improves memory deficit in mice fed a high-fat diet. Diabetes 2012;61:1444-1454.

36 Witte AV, Kerti L, Margulies DS, Flöel A: Effects of resveratrol on memory performance, hippocampal functional connectivity, and glucose metabolism in healthy older adults. J Neurosci 2014;34:7862-7870.

-37 Atri A, O’Brien JL, Sreenivasan A, Rastegar S, Salisbury S, DeLuca AN, O’Keefe KM, LaVioloette PS, Rentz DM, Locascio JJ, Sperling RA: Test-retest reliability of memory task functional magnetic resonance imaging in Alzheimer disease clinical trials. Arch Neurol 2011;68:599-606.

-38 Clément F, Belleville S: Test-retest reliability of fMRI verbal episodic memory paradigms in healthy older adults and in persons with mild cognitive impairment. Hum Brain Mapp 2009;30:4033-4047.

-39 Gustafson D, Rothenberg E, Blennow K, Steen B, Skoog I: An 18-year follow-up of overweight and risk of Alzheimer disease. Arch Intern Med 2003;163:1524-1528.

40 Lovestone S, Smith U: Advanced glycation end products, dementia, and diabetes. Proc Natl Acad Sci U S A 2014; 111:4743-4744.

-41 Whalley LJ, Deary IJ, Starr JM, Wahle KQ, Rance KA, Bourne VJ, Fox HC: n-3 Fatty acid erythrocyte membrane content, APOE varepsilon4, and cognitive variation: an observational follow-up study in late adulthood. Am J Clin Nutr 2008;87:449-454. 